\title{
REDUCED ORDER MODEL OF CIRCULAR FLANGE-BOLTED CONNECTION AND ITS APPLICATION TO DYNAMIC SUBSTRUCTURING OF TELECOMMUNICATION TOWERS
}

\author{
Bartlomiej D. Blachowski ${ }^{1}$, Witold Gutkowski ${ }^{2}$ and Piotr Wisniewski ${ }^{1}$ \\ ${ }^{1}$ Institute of Fundamental Technological Research \\ Pawinskiego 5b, 02-106 Warsaw, Poland \\ e-mail: \{bblach, pwisn $\} @$ ippt.gov.pl \\ ${ }^{2}$ Institute of Mechanized Construction and Rock Mining \\ Racjonalizacji 6/8, 02-673 Warsaw, Poland \\ wgutkow@ippt.gov.pl
}

Keywords: Reduced order modeling, Circular flange-bolted connections, Dynamic substructuring, Nonlinear contact and friction effects, Telecommunication towers.

\begin{abstract}
.
The paper deals with a nonlinear analysis of a tall tower, with Circular Flange Bolted Connections $(C F B C)$, in which friction and contact effects are taken into account. Due to these nonlinearities, a detailed dynamic model of the whole structure would lead to very complex computational problem, unable for practical solutions. To overcome these difficulties a reduced order model of $C F B C$ is proposed. Such a model enables the simulation of the whole tower including nonlinearities in connections. The paper is illustrated with an example of model reduction and dynamic calculations for a contemporary telecommunication tower. The tower is assembled of 4 truss segments, of triangular cross section, interconnected with CFBCs. Finally, the influence of the number of modes, included in the reduced order model, on the accuracy and computational effect, is discussed.
\end{abstract}




\section{INTRODUCTION}

Telecommunication towers, guyed masts and other engineering structures are made of circular hollow profiles. Tower sections welded in the factory, are on site, assembled with circular flange bolted connection (CFBC) shown on Fig. 1.

In a global analysis of tall structures, the mechanical properties of CFBCs are often neglected, under assumption, that they are rigid. However, how it was shown by Blachowski and Gutkowski [1], the influence of the their flexibility on the dynamic response of the structures can not be neglected.

According to the recent trends in structural health monitoring (SHM) it is necessary, among others, to monitor and detect structural damages. Two main methods in SHM can be recognized: local methods [2,3,4] and global ones [5-6]. In local methods connections are monitored directly at the vicinity of the damage. However, the local data don't give information about the health of the whole structure. In the global methods we are monitoring the response of the whole structure by measuring only selected quantities, such as accelerations of assumed structural points. The global methods require precise FE model of the entire structure, which may require a significantly great numerical effort. It is then useful, in such cases, to apply, either simplified [7] or detailed joint models [8]. In the former, connections are considered in a, more or less, approximate way. The latter require sophisticated computer clusters analyzing global behavior of the entire structure. Practical reasons push us, then towards reduced order modeling (ROM).

In this work, a reduced order model (ROM) of the circular flange-bolted connection is presented. The model takes into account the inherent nonlinearity of the connections, consisting of contact and friction forces between adjacent circular flanges and pre-tensioning of bolts. Having the model, describing behavior of CFBC, it is possible to work on a dynamic model of the whole tower. For that purpose the Ritz Vectors as a reduction basis [10] is applied. The paper is illustrated with an example of ROM applied to dynamic simulation of a contemporary telecommunication tower. The tower is assembled of 4 truss segments, of triangular cross section, jointed with CFBCs. One of the connections is assumed to be damaged. Finally, the influence of the number of modes, included in the ROM, on the accuracy and computational effort, is discussed.

\section{SUBSTRUCTURING OF A TELECOMUNICATION TOWER}

The tower (Fig.1) is composed of two kinds substructures: truss/frame segments (segment substructure) and circular flange connections (connection substructure). One the flange is welded to the segment end. Brought on the side, the segments are assembled together with, bolts passing through the adjacent flanges. Bolts are pre-stressed to given forces, assumed by a designer. A segment with welded flanges constitutes a structure made of a linear material. However, the assembled tower must be considered as a nonlinear system. This is due the contact and friction forces taking place between flanges and bolts.

In Fig 2, a CFBC consisting of pre-tensioned bolts and contacting flanges is presented. 

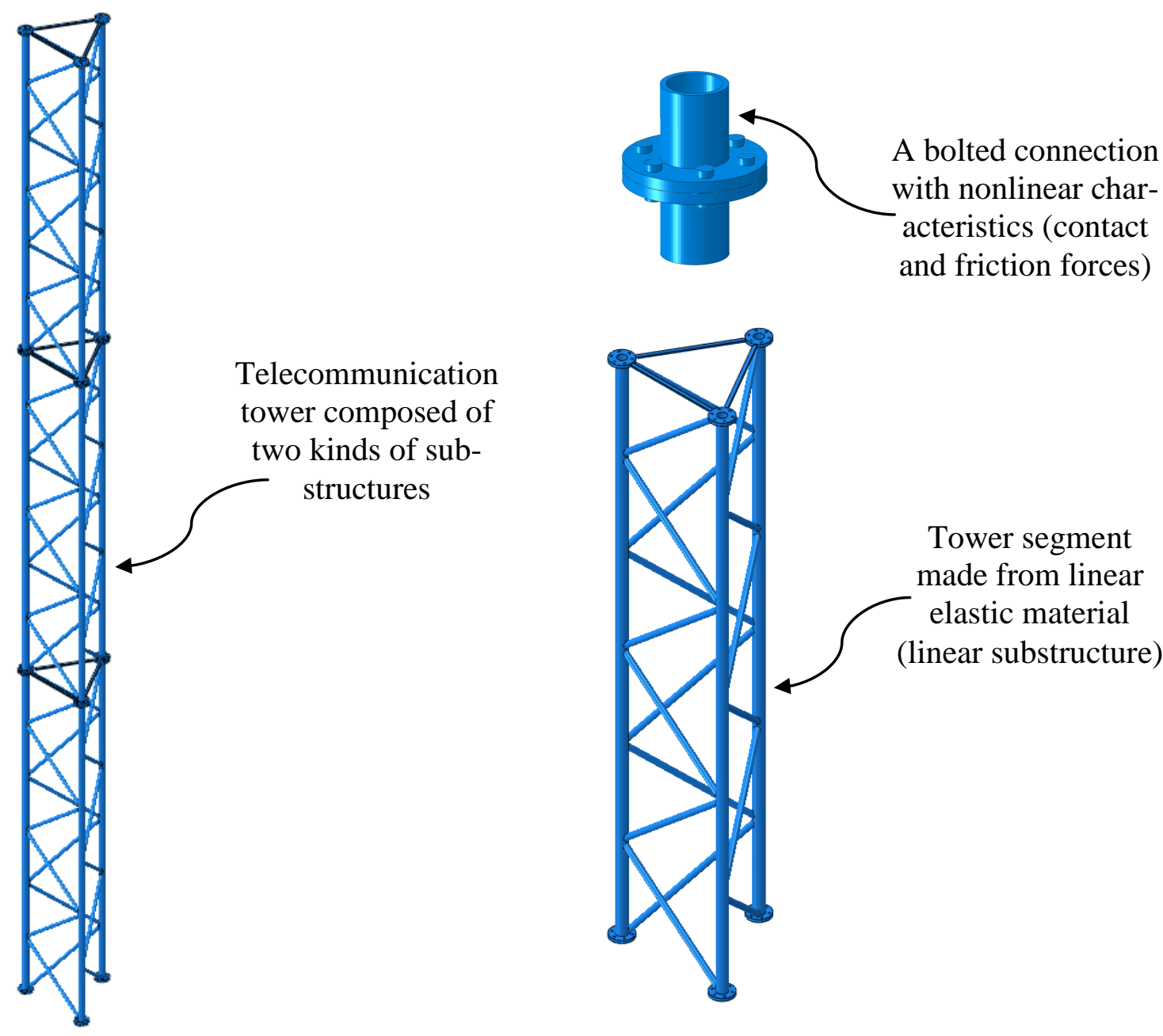

Telecommunication tower composed of two kinds of sub-

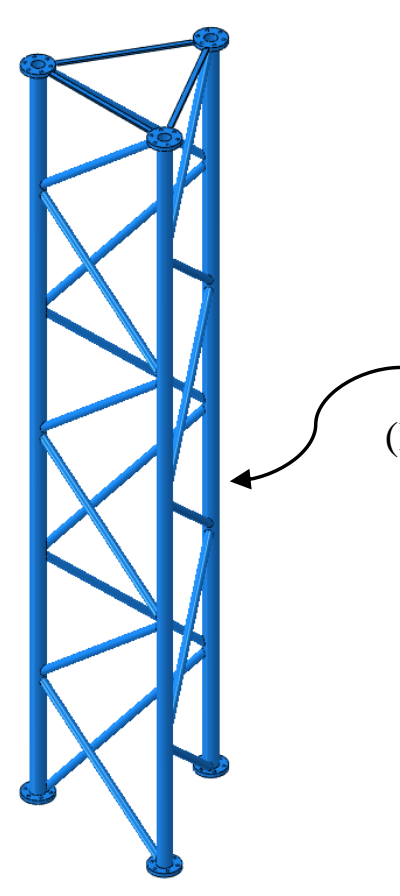

Tower segment made from linear elastic material (linear substructure)

Figure 1. Telecommunication tower and its substructures

\section{REDUCED MODEL OF A CFBC}

In modeling the CFBC, isoparametric elements of 3D FEM are applied. The model is created using a substructuring technique [9]. Each of the connection components are modeled separately, and then joined together, they constitute a model of one structure.

After defining the stiffness matrices of the components, the model contact between them is performed, leading to the equilibrium equations of the $\mathrm{CFBC}$ in the form:

$$
\begin{cases}\mathbf{K}^{(c)} \mathbf{u}^{(c)}=\mathbf{f}^{(c)}+\mathbf{g}^{(c)}\left(\mathbf{u}^{(c)}\right) & \text { - Compatibility condition } \\ \mathbf{B} \mathbf{u}^{(c)}=\mathbf{0} & \text { - Equilibrium of contact (interface) forces } \\ \mathbf{L}^{\mathbf{T}} \mathbf{g}^{(c)}\left(\mathbf{u}^{(c)}\right)=\mathbf{0} & \end{cases}
$$

where : $\mathbf{K}^{(\mathrm{c})}$ is the connection stiffness matrix, $\mathbf{f}^{(c)}$ is the vector of external forces applied to the connection, and $\mathbf{g}^{(c)}$ contains interface, contact and friction forces. 


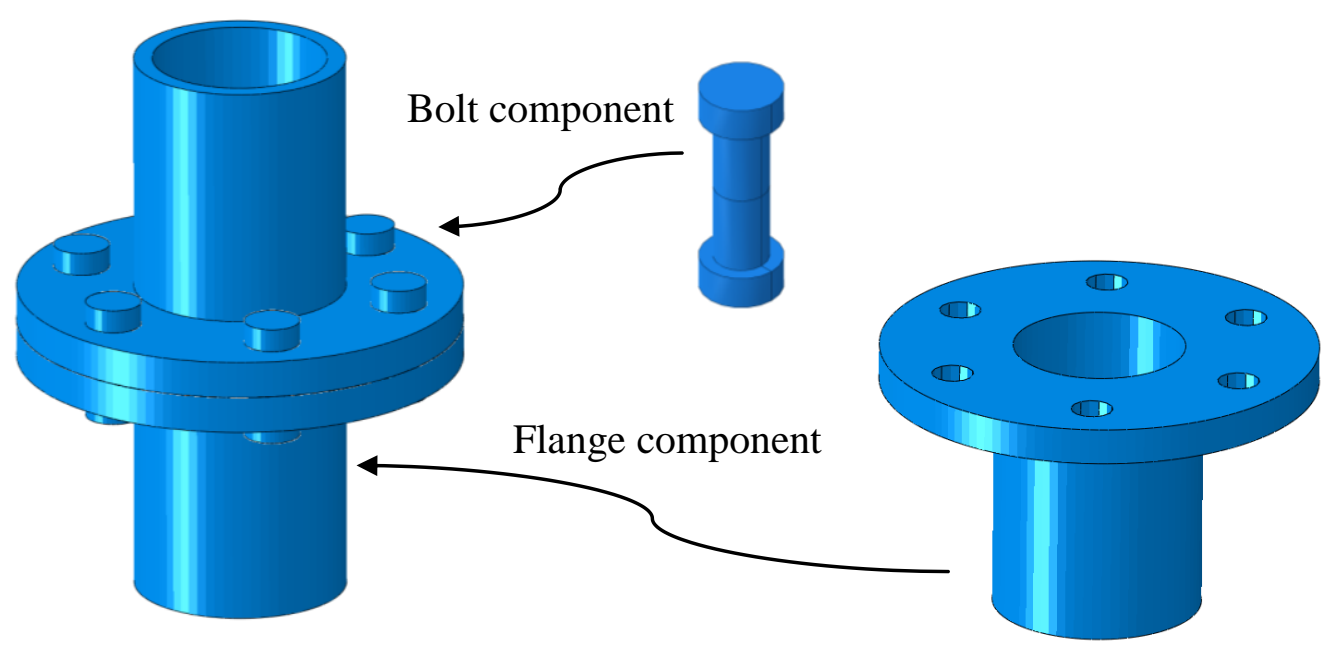

Figure 2. Circular flange-bolted connection and its components

Now, the simulation finding the rigidity of the CFBC is performed with axial forces $f$ applied to the ends of the flanges. The connection is assumed to be composed of $30 \mathrm{~mm}$ thick flanges and bolts of M20. Each connection is welded to the tubes of $114.3 \mathrm{~mm}$ diameter and wall thickness of $10 \mathrm{~mm}$.

The analysis is performed for four different values of pre-stressing bolt force; namely: 100; 80; 60; $40 \mathrm{kN}$. The force-deformation diagrams for all mentioned cases are presented in Fig 3. The above diagrams allow representing the particular CFBC by nonlinear elastic elements, which will be applied in the reduced tower model. If connections are damaged they can be represented by the mentioned above nonlinear element but of the much smaller stiffness.

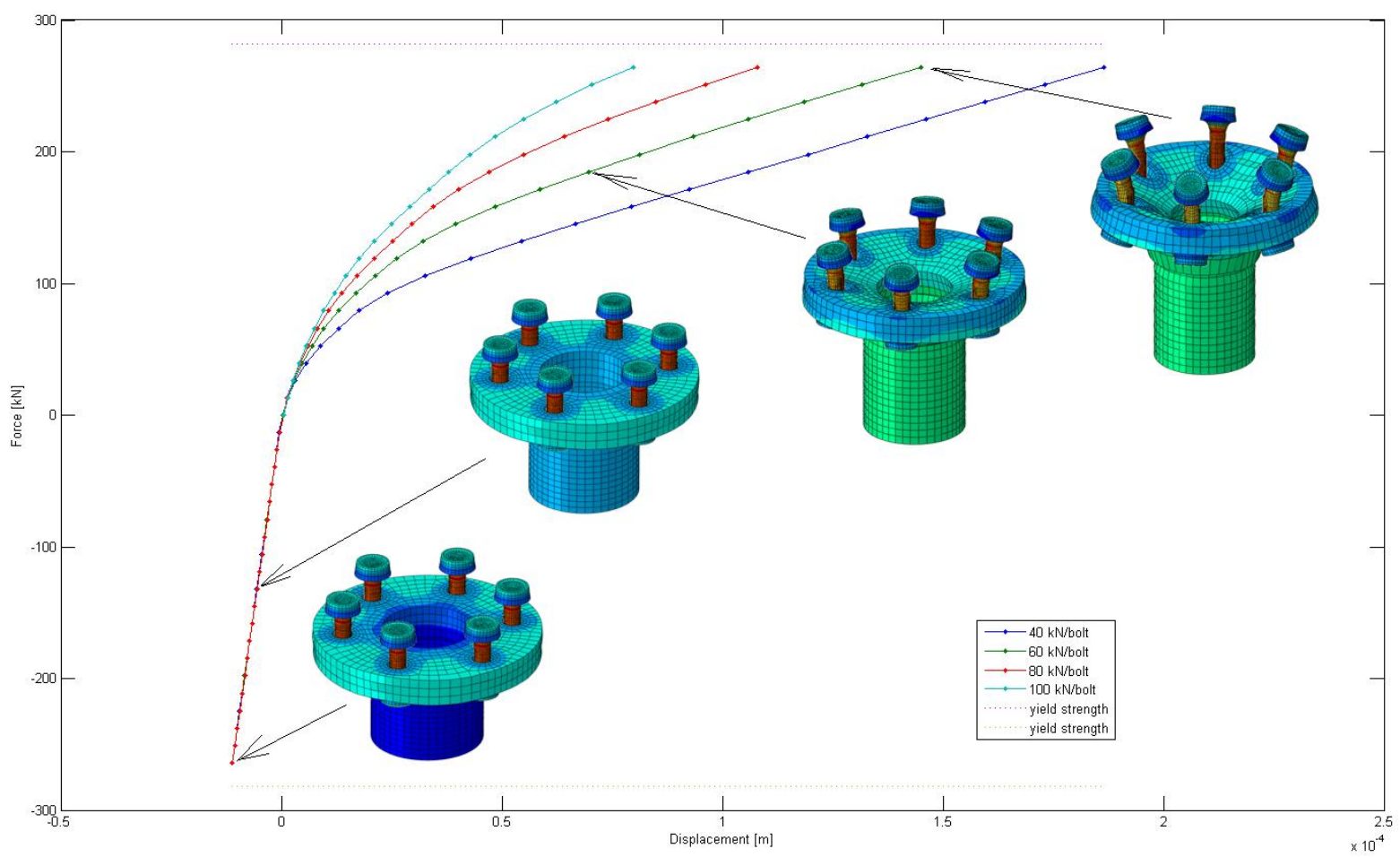

Figure 3. Force-deformation relation for CFBC with four different bolt pre-tension 


\section{RESPONSE OF AN ASSAMBLED TELECOMUNICATION TOWER TO EXTERNAL DYNAMIC FORCES}

Equations of motion for a nonlinear structure have the following form

$$
\mathbf{M} \ddot{\mathbf{q}}(t)+\mathbf{r}(\mathbf{q}(t), \dot{\mathbf{q}}(t))=\mathbf{f}^{e x t}(t)
$$

where: $\mathbf{M}$ - mass matrix, $\mathbf{q}(t), \dot{\mathbf{q}}(t), \ddot{\mathbf{q}}(t)$ are respectively displacement, velocity and acceleration vectors, $\mathbf{r}(\mathbf{q}(t), \dot{\mathbf{q}}(t))$ - nonlinear internal forces including damping and elastic forces, $\mathbf{f}^{\text {ext }}(t)$ - external force and $t$ denotes time. The above equations should be considered together with compatibility and interface equilibrium conditions, similarly to equations (1).

Taking into account the nonlinear forces occurring at the tower connections, equations of motion for $i$-th segment (substructure) can be presented as follows

$$
\left[\begin{array}{ccc}
\mathbf{M}_{u u} & \mathbf{M}_{u m} & \mathbf{0} \\
\mathbf{M}_{m u} & \mathbf{M}_{m m} & \mathbf{M}_{m l} \\
\mathbf{0} & \mathbf{M}_{l m} & \mathbf{M}_{l l}
\end{array}\right]^{(i)}\left[\begin{array}{c}
\ddot{\mathbf{q}}_{u} \\
\ddot{\mathbf{q}}_{m} \\
\ddot{\mathbf{q}}_{l}
\end{array}\right]^{(i)}+\left[\begin{array}{ccc}
\mathbf{K}_{u u} & \mathbf{K}_{u m} & \mathbf{0} \\
\mathbf{K}_{m u} & \mathbf{K}_{m m} & \mathbf{K}_{m l} \\
\mathbf{0} & \mathbf{K}_{l m} & \mathbf{K}_{l l}
\end{array}\right]^{(i)}\left[\begin{array}{c}
\mathbf{q}_{u} \\
\mathbf{q}_{m}^{(i)} \\
\mathbf{q}_{l}
\end{array}\right]^{\left(\mathbf{f}_{u}^{N L}\right.}+\left[\begin{array}{c}
\mathbf{0} \\
\mathbf{f}_{l}^{N L}
\end{array}\right]^{(i)}=\left[\begin{array}{c}
\mathbf{f}_{u}^{e x t} \\
\mathbf{f}_{m}^{e x t} \\
\mathbf{f}_{l}^{e x t}
\end{array}\right]^{(i)}
$$

where: subscripts $u, l$ denote upper and lower nodes. The subscript $m$ denotes all other nodes. With above notation the equations (3) can be presented shortly as

$$
\mathbf{M}^{(i)} \ddot{\mathbf{q}}^{(i)}+\mathbf{K}^{(i)} \mathbf{q}^{(i)}+\mathbf{f}^{N L^{(i)}}=\mathbf{f}^{\text {ext }}{ }^{(i)} \quad i=1,2, \ldots, n_{S},
$$

where: $\mathbf{M}^{()}, \mathbf{K}^{(i)}$ - mass and stiffness matrices of $i$-th tower segment, $\mathbf{f}^{N L(i)}$ - nonlinear forces coming from contact and friction with neighboring segments, $n_{S}$ - number of the tower segments. The above set of equations is now solved using direct integration approach.

\section{DIRECT INTEGRATION OF NON-LINEAR MODEL OF THE TOWER}

Combining all $n_{s}$ equations (4) we come to the full order mode (FOM) of the tower

$$
\mathbf{M}_{F O M} \ddot{\mathbf{q}}_{F O M}+\mathbf{K}_{F O M} \mathbf{q}_{F O M}+\mathbf{f}_{F O M}^{N L}=\mathbf{f}^{e x t}
$$

Applying substructuring method for the contact forces, we can represent stiffness matrix and nonlinear internal forces in the following form
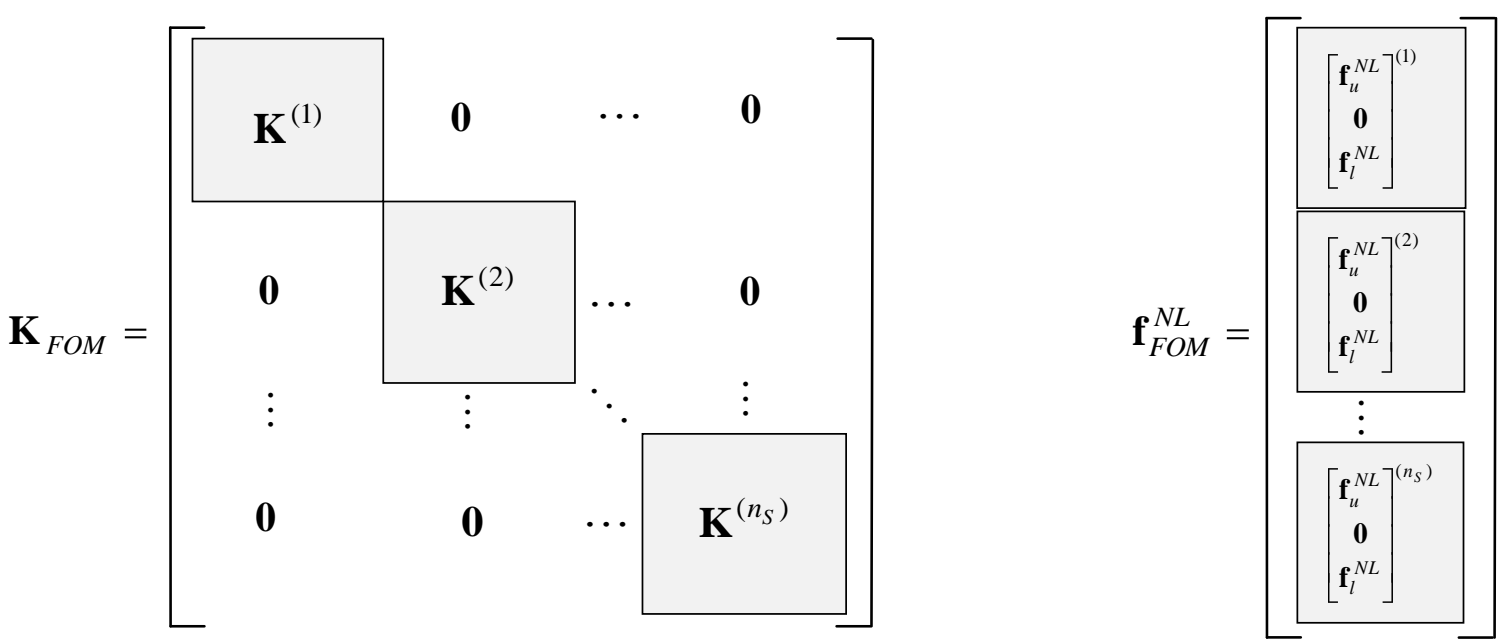

where $\mathbf{f}_{F O M}^{N L}$ represents terms, coupling stiffness matrices of elements in contact. 


\section{REDUCED ORDER MODEL OF THE TOWER BASED ON SUBSPACE PROJECTION}

Let now reduce the FOM to a simpler model. It consists in dividing the dynamic problem in two steps. The first one is based on nonlinear static analysis giving the response of the tower to mean (static) value of the dynamic loading. In the second step, we are reducing the system of dynamic equations by applying Ritz vector approach. In this way we obtain a smaller number of coupled equations, representing dynamic modes of the vibrating tower.

Based on the above assumption we formulate the problem as follows:

Step 1

We perform static analysis with the following equation

$$
\mathbf{K}_{0}\left(\mathbf{q}_{0}\right) \mathbf{q}_{0}=\mathbf{f}_{0}{ }^{\text {ext }}
$$

Step 2

We reduce the number of dynamic equations starting with static response $\mathbf{v}_{1}=\mathbf{q}_{0}$, as the first vector of the reduced basis. Next, we apply the Gram-Schmidt orthogonalization for finding whole set of vectors $\mathbf{V}=\left[\mathbf{v}_{1}, \mathbf{v}_{2}, \ldots, \mathbf{v}_{n_{R}}\right]$. This way we get a reduced order model $(R O M)$

$$
\ddot{\eta}_{F O M}+\mathbf{K}_{R O M} \eta_{R O M}+\mathbf{f}_{R O M}^{N L}=\mathbf{V}^{T} \tilde{\mathbf{f}}^{e x t}
$$

where

$$
\begin{aligned}
& \mathbf{V}^{T} \mathbf{M}_{F O M} \mathbf{V}=\mathbf{I} \\
& \mathbf{K}_{R O M}=\mathbf{V}^{T} \mathbf{K}_{0} \mathbf{V} \\
& \mathbf{f}_{R O M}^{N L}=\mathbf{V}^{T} \mathbf{f}_{F O M}^{N L}
\end{aligned}
$$

and $\tilde{\mathbf{f}}^{\text {ext }}$ denotes fluctuating part of external loading and $\eta_{R O M}$ modal amplitude vector.

\section{NUMERICAL EXAMPLE - SIMULATION OF THE TOWER DYNAMIC RESPONSE WITH DAMAGED CONNECTIONS}

The tower is assembled of 4 truss segments, of triangular cross section, interconnected with CFBCs. The dimension of the triangular cross section of the tower's segment is $1.2 \mathrm{~m}$, and the height of the segment is $6 \mathrm{~m}$. The segment is made of $114.3 \mathrm{~mm}$ diameter tubes, having $8 \mathrm{~mm}$ wall thickness. CFBCs are composed of two $3 \mathrm{~cm}$ thick circular flanges, assembled with 6 pre-tensioned bolts of $20 \mathrm{~mm}$ diameter. Bolts in all connections are pre-tensioned, with the $400 \mathrm{Nm}$ torque. It is assumed that one connection located between first and second truss segment (Figure 4), may be partially damaged. It means, that their stiffness for tension is smaller than those of healthy ones. 


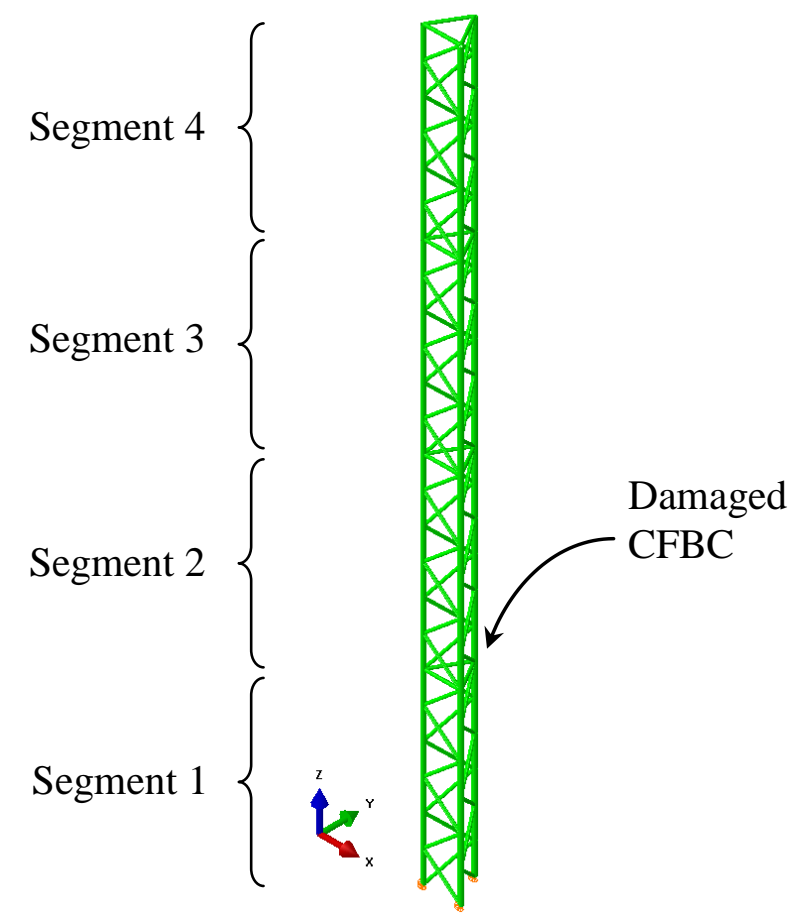

Figure 4. Telecommunication tower under investigation.

As it was mentioned in chapter 3, damaged CFBC exhibits strongly nonlinear behavior. It can be observed from Fig 3, that compression stiffness can be few orders of magnitude higher than for tension. For that reason, two cases of the dynamic response of the tower with a damaged connection are considered. It is assumed that in both of them, forces acting on the tower have the time history, as shown in Fig 5 and are composed of two parts. The first one is the long-term wind pressure causing static deformation of the tower. The second one is the instantaneous wind gusts, inducing oscillations of the tower.

Depending on the direction of the forces acting on the tower, the damaged CFBC is either in compression, or in tension (Fig 6).

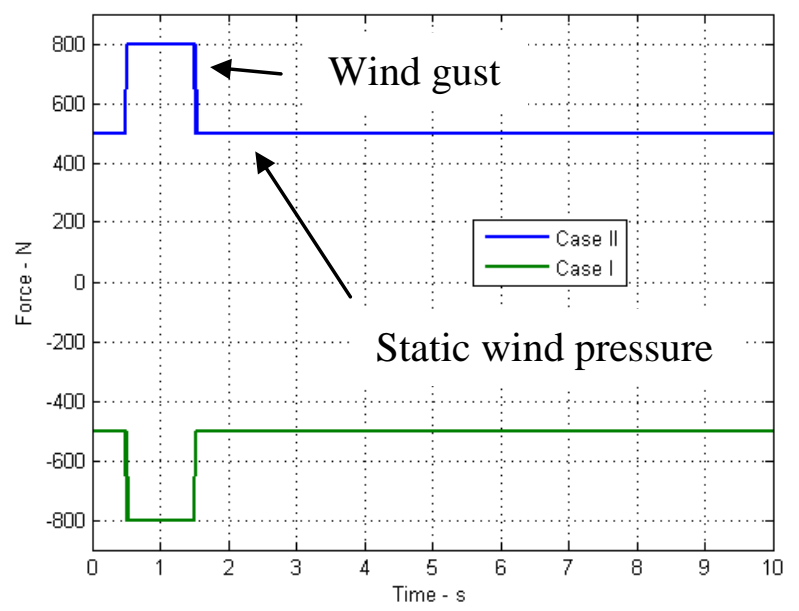

Figure 5. Time history of the forces in dynamic analysis 


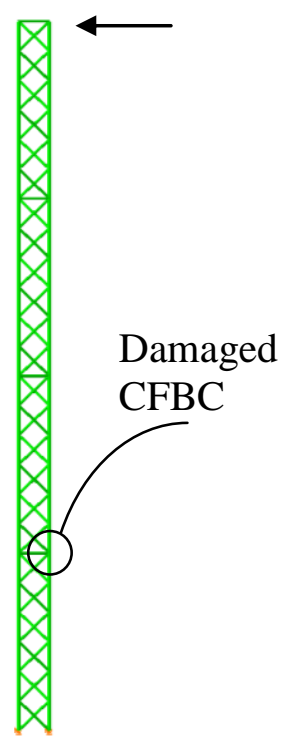

Case I: $\mathrm{CFBC}$ is in tension
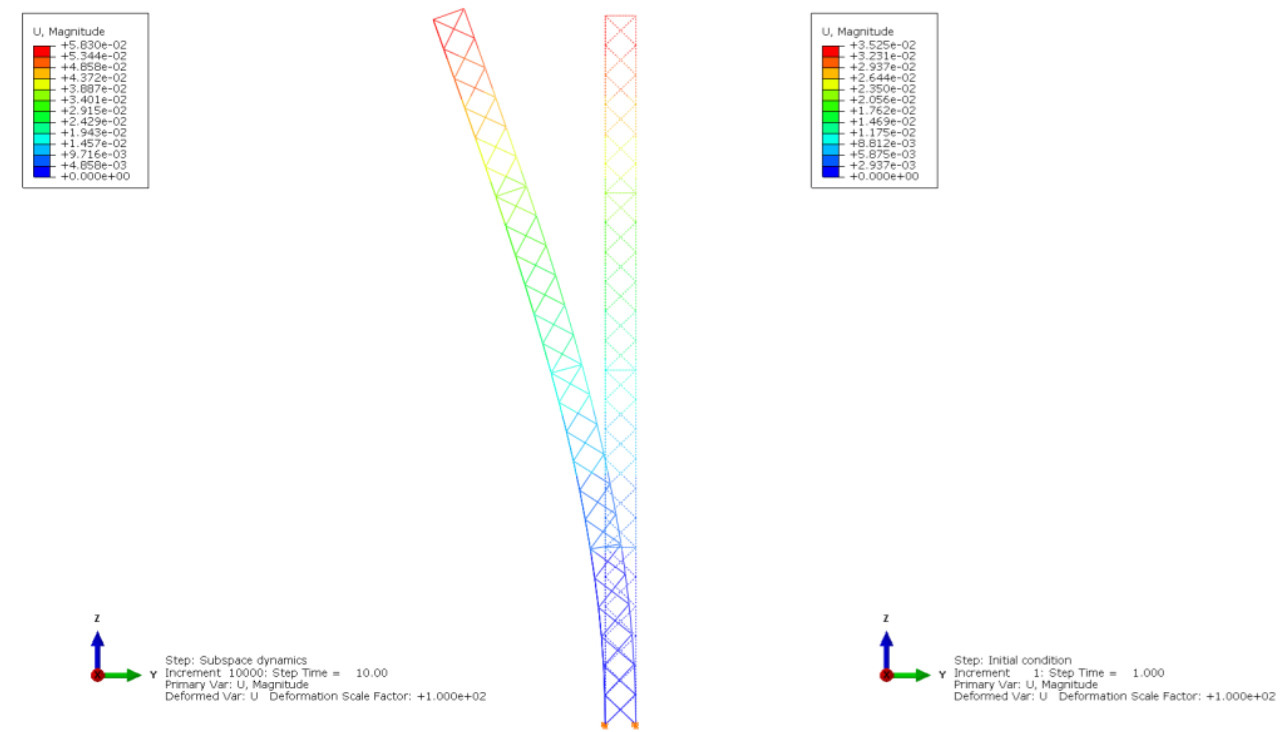

Case II: CFBC is in compression

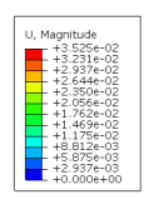

Damaged

CFBC
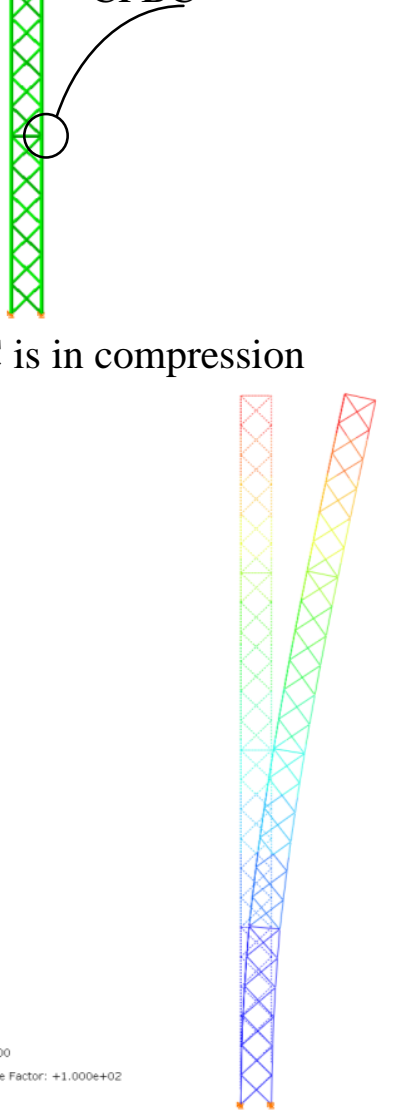

Figure 6. The damaged CFBC in tension (case I) and in compression (case II).

The tower deformation is magnified 100 times.

Unsymmetrical response of the tower under static wind pressure, depending on its direction can be observed on Fig 6. This is caused by much lower stiffness of CFBC in tension, than in compression.

After determining initial static deformation of the tower, the natural frequencies and mode shapes for both cases are discussed.

The first three mode shapes for both discussed cases are shown in Fig 7. One can observe differences in the corresponding natural frequencies. Comparing first frequencies for both cases, we find that first natural frequency for the case II is smaller than in the case I. 
Mode 1

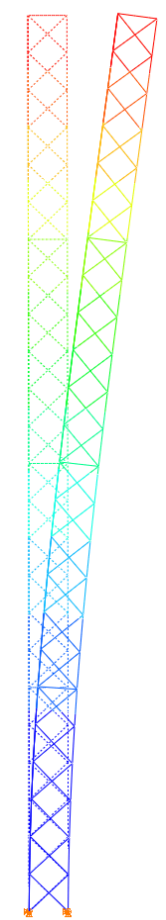

Case I

Case II
$1.6267 \mathrm{~Hz}$

$1.5508 \mathrm{~Hz}$
Mode 2

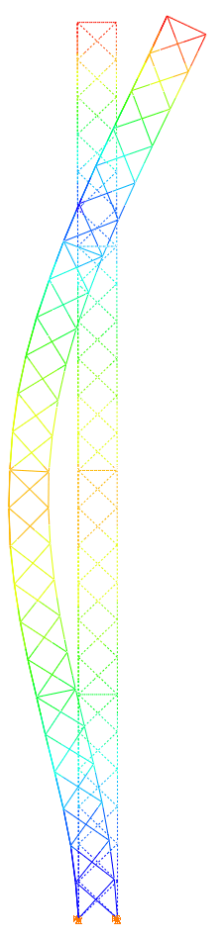

$9.6388 \mathrm{~Hz}$

$9.6182 \mathrm{~Hz}$
Mode 3

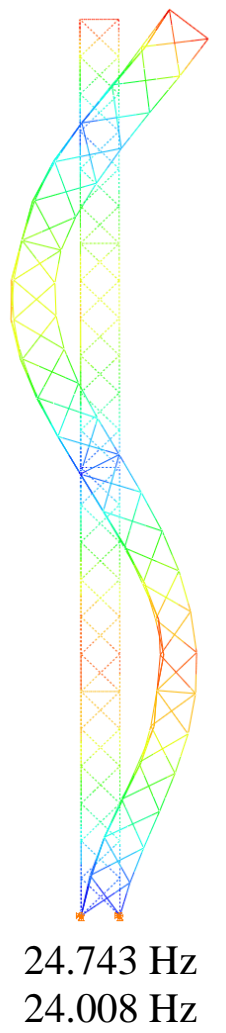

Figure 7. Comparison of the natural frequencies in both cases.

After having determined the mode shapes of the tower with damaged CFBC, the response time history of the tower is found and shown on Figures 8 and 9.

Time responses for both cases have been obtained using techniques described in chapters 5 and 6, namely: Direct Integration and Reduced Order Model. In the latter one, a different number of modes are included into analysis, showing their influence on accuracy of calculations (Figure 8 and 9).

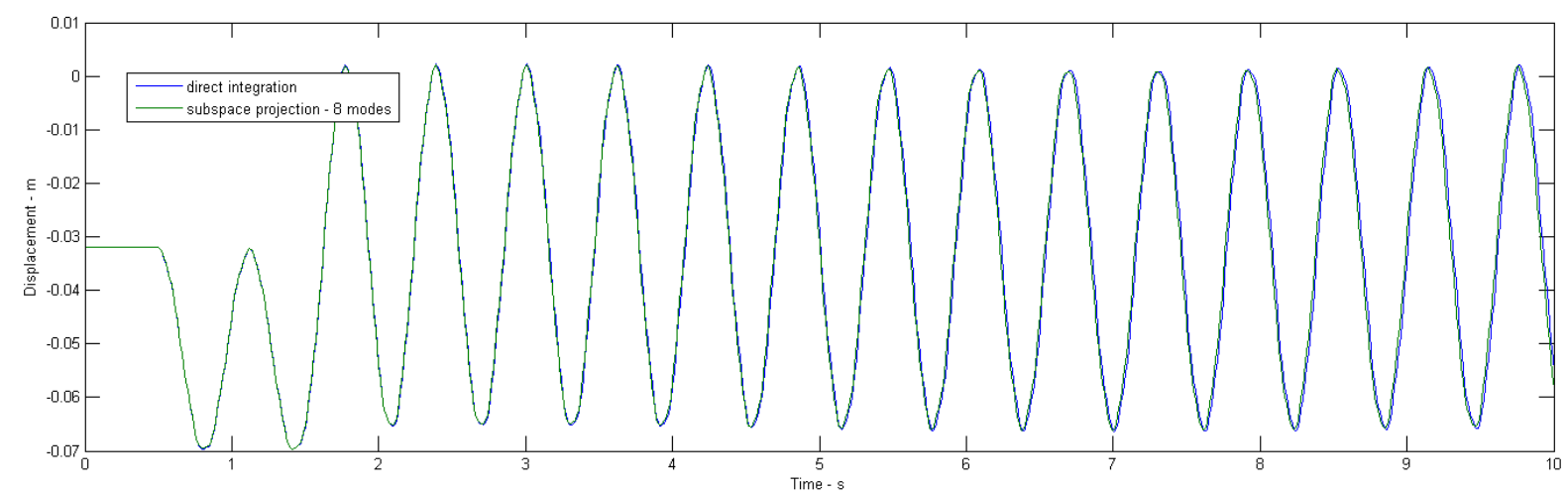

Figure 8. Displacement of the top of the tower (Case I). 


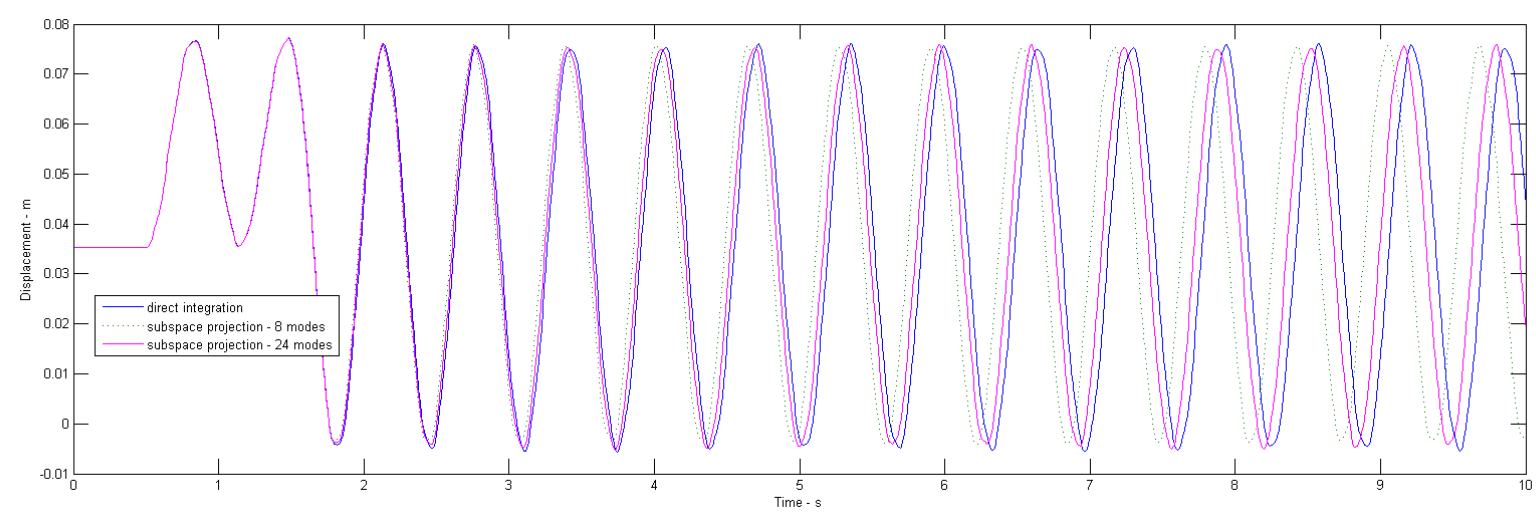

Figure 9. Displacement of the top of the tower (Case II).

Comparing Figures 8 and 9 we conclude that 8 modes are enough to estimate the tower response, in the Case I. The displacement responses obtained with the aid of both methods are almost the same. This is showing that with ROM, significant computational efforts can saved. However, this is not the case, when CFBC is in compression. Up to 24 modes have to be used, in order to obtain better estimation of the direct integration solution. Using only 8 modes in ROM, it is insufficient to estimate the response of the structure with assumed accuracy.

Finally, in the figure 10, the Fourier transform of displacements in both cases is compared. It confirmed, that the in the Case II structure vibrates with fundamental frequency, lower than in the Case I.

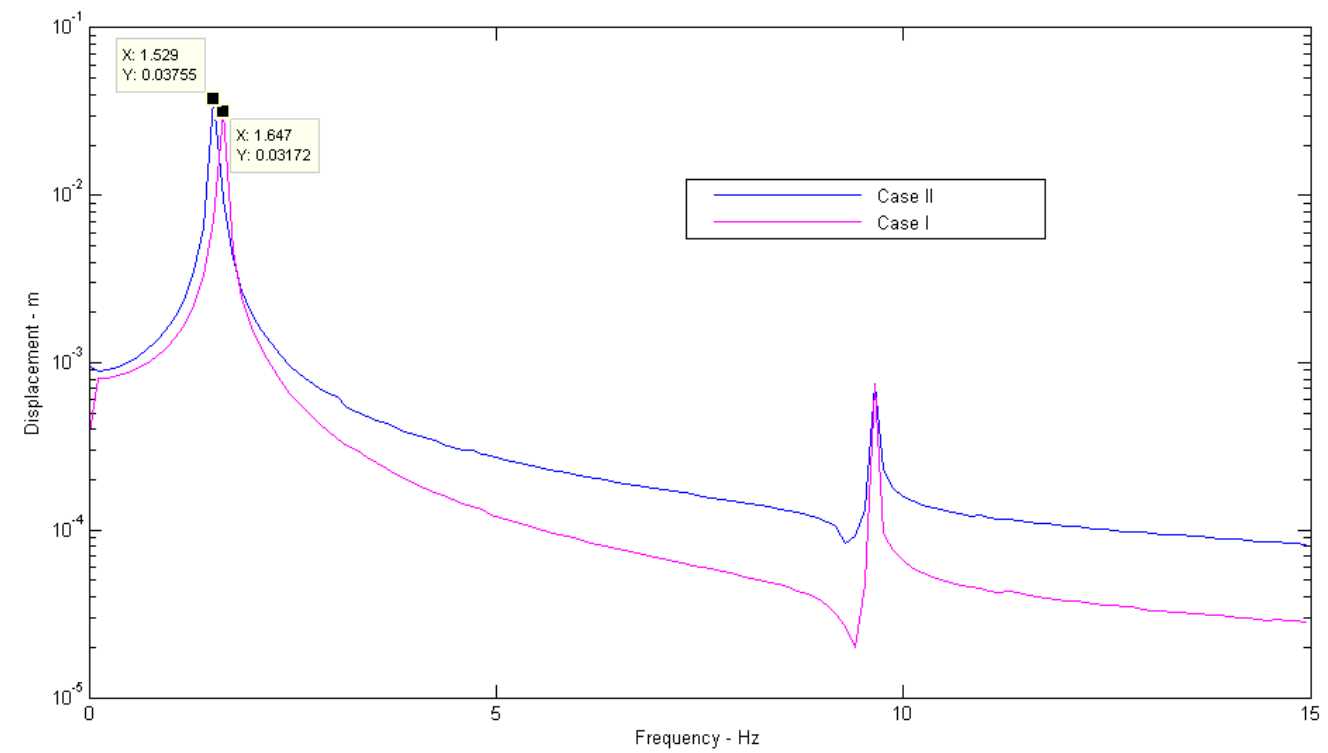

Figure 10. Fourier transform of the displacements.

\section{CONCLUSIONS}

A Reduced Order Model of vibrating telecommunication tower is presented. The model is composed of two parts. In the first one, applying substructuring approach, the reduced model of a CFBC, including contact and friction forces, is discussed (Section 3). The connection composed of flanges and bolts is here reduced to a nonlinear elastic element. 
In the second part, tower segments are considered as substructures, and then the CFBC structure is replaced by mentioned above nonlinear elastic elements (Section 6). This approach allows diminishing the numerical efforts by reducing the model to few important modal shapes. The direct integration of non-linear, whole model of the tower (Section 5), in turn, gives a view how much results obtained by the reduced model differs from exact ones. This can be observed in the numerical example (Section 7).

Moreover, in the example, a notion of a damaged connection is introduced. The damage is represented by an elastic nonlinear element of stiffness, several times smaller than stiffness of healthy connections. The tower is subjected to dynamic wind forces, which are decomposed in long-term wind pressure, causing static deformation of the tower and instantaneous wind gusts, inducing oscillations of the tower.

The differences in the deformations of a tower, with all healthy CFBCs, and one damaged don't look very significant from the structural point of view. Nevertheless, in some cases, when the damage consists in failure of one or more bolts the stress in nonlinear connection can significantly increase.

\section{ACKNOWLEDGEMENTS}

The authors are grateful for financial support from the National Science Centre of Poland (grant N N501 0494 40).

\section{REFERENCES}

[1] B.Blachowski, W.Gutkowski, Revised assumptions for monitoring and control of $3 D$ lattice structures. 11th Pan-American Congress of Applied Mechanics PACAM XI, Foz do Iguassu, Brasil, 2010.

[2] F. Amerini, and M. Meo., Structural health monitoring of bolted joints using linear and nonlinear acoustic/ultrasound methods. Structural Health Monitoring - An International Journal, 10(6), 659-672, 2011.

[3] I. Pavelko, V. Pavelko, S. Kuznecovs, I. Ozolins, Bolt-Joint Structural Health Monitoring by the Method of Electromechanical Impedance. Aircraft Engineering and Aerospace Technology, 86(3), 207-214. ISSN 0002-2667. Available from: doi:10.1108/AEAT-01-2013-0006, 2014.

[4] T. Wang, G. Song, S. Liu, Y. Li, and H. Xiao, Review of Bolted Connection Monitoring, International Journal of Distributed Sensor Networks, (2013), Article ID 871213, 8 pages, http://dx.doi.org/10.1155/2013/871213, 2013.

[5] J. N. Yang; Y. Xia; and C.H. Loh, Damage Identification of Bolt Connections in a Steel Frame, ASCE Journal of Structural Engineering, 140(3), 04013064, 2014.

[6] K. He, W.D. Zhu, A vibration-based structural damage detection method and its applications to engineering structures, International Journal of Smart and Nano Materials, 09/2011, 2:3, 194-218, DOI: 10.1080/19475411.2011.594105, 2011. 
[7] Y. Luan, Z.Q. Guan, G.D. Cheng, S. Liu, A simplified nonlinear dynamic model for the analysis of pipe structures with bolted flange joints, Journal of Sound and Vibration, 331, 325-344, 2012.

[8] V. Roulet, L. Champaney, P.A. Boucard, A parallel strategy for the multiparametric analysis of structures with large contact and friction surfaces, Advances in Engineering Software, 42(6), 347-358, 2011.

[9] D. de Klerk, D. J. Rixen, and S. N. Voormeeren, General Framework for Dynamic Substructuring: History, Review, and Classification of Techniques, AIAA Journal, 46(5), 2008.

[10] R.K. Kapani and C. Byun, Reduction methods based on eigenvectors and Ritz vectors for nonlinear transient analysis, Computational Mechanics, 11, 65-82, 1993. 